\title{
ON THE DISTRIBUTION OF EXTREMAL POINTS OF GENERAL CHEBYSHEV POLYNOMIALS
}

\author{
ANDRÁS KROÓ AND FRANZ PEHERSTORFER
}

\begin{abstract}
For a linear subspace $\mathscr{U}_{n}=\operatorname{span}\left[\varphi_{1}, \ldots, \varphi_{n}\right]$ in $C[a, b]$ we introduce general Chebyshev polynomials as solutions of the minimization problem $\min _{a_{i}}\left\|\varphi_{n}-\sum_{i=1}^{n-1} a_{i} \varphi_{i}\right\|_{C}$. For such a Chebyshev polynomial we study the distribution of its extremal points (maximum and minimum points) in terms of structural and approximative properties of $\mathscr{U}_{n}$.
\end{abstract}

\section{INTRODUCTION}

Consider a sequence of linear subspaces $\mathscr{U}_{1}, \mathscr{U}_{2}, \ldots, \mathscr{U}_{n}, \ldots, \operatorname{dim} \mathscr{U}_{n}=n$ in the real space $C[a, b]$. For a basis $\left[\varphi_{1}, \ldots, \varphi_{n}\right]$ in $\mathscr{U}_{n}$ we can set the minimization problem:

$$
\min _{a_{i}}\left\|\varphi_{n}-\sum_{i=1}^{n-1} a_{i} \varphi_{i}\right\|_{C},
$$

where $\|f\|_{C}$ denotes the usual supremum norm. (Note that we do not assume that $\mathscr{U}_{n} \subset \mathscr{U}_{n+1}$ and that the choice of the basis in $\mathscr{U}_{n}$ is arbitrary.)

For the classical case of algebraic polynomials the solution of (1) is given by the Chebshev polynomial of first kind. In accordance with this we shall call a solution $T_{n}=\varphi_{n}-\sum_{i=1}^{n-1} a_{i}^{*} \varphi_{i}$ of (1) a general Chebyshev polynomial with respect to the basis $\left[\varphi_{1}, \ldots, \varphi_{n}\right]$ of $\mathscr{U}_{n}$. (Note that in general the solution of (1) is not unique.) Apart from the case of algebraic (or trigonometric) polynomials not much is known about the distribution of the points where a solution $T_{n}$ of (1) attains its maximal or minimal value. (In what follows we shall call such points extremal points of $T_{n}$.)

M. v. Golitschek raised the question whether the extremal points of $T_{n}$ are dense in $[a, b]$ as $n \rightarrow \infty$, if we assume that $\mathscr{U}_{n}$ is a $T$-space, $n=1,2, \ldots$. (Recall that $\mathscr{U}_{n}$ is called a $T$-space if the elements of $\mathscr{U}_{n}$ have at most $n-1$ zeros on $[a, b]$.) It turned out that the $T$-property is not enough to guarantee the density but adding the assumption $\mathrm{Cl}\left(\bigcup_{n=1}^{\infty} \mathscr{U}_{n}\right)$ has finite codimension in $C[a, b]$ we can obtain the desired result. Moreover the assumption that $\mathscr{U}_{n}$ is a

Received by the editors July 12, 1988 and, in revised form, September 5, 1989.

1980 Mathematics Subject Classification (1985 Revision). Primary 41A50.

Key words and phrases. Chebyshev polynomials, density of extremal points.

The paper was written during the second author's visit at the Mathematical Institute of the Hungarian Academy of Sciences. Research supported by Hungarian National Foundation for Scientific Research Grant No. 1801. 
$T$-space can be essentially weakened and in addition we can obtain quantitative estimates of the density.

The paper is organized as follows. First we formulate and prove our main positive results. Then we proceed with giving some applications. Finally, in the third part of the paper we provide different counterexamples showing that the assumptions we impose in order to get positive results are in general necessary.

\section{Positive RESULTS}

Let us give some additional notations and definitions. For linear subspaces $\mathscr{U}_{n}(n=1,2, \ldots)$ in $C[a, b]$, a function $f \in C[a, b]$ and a set $G \subset C[a, b]$ we set

$$
\begin{aligned}
& E\left(f, \mathscr{U}_{n}\right)=\inf \left\{\left\|f-p_{n}\right\|: p_{n} \in \mathscr{U}_{n}\right\}, \\
& E\left(G, \mathscr{U}_{n}\right)=\sup \left\{E\left(f, \mathscr{U}_{n}\right): f \in G\right\} .
\end{aligned}
$$

Furthermore,

$$
\begin{aligned}
W^{1} & =\left\{f \in C[a, b]:\|f\|_{C} \leq 1, \omega(f, h) \leq \frac{h}{b-a}\right\}, \\
\mathscr{U} & =\left\{f \in C[a, b]: \lim _{n \rightarrow \infty} E\left(f, \mathscr{U}_{n}\right)=0\right\},
\end{aligned}
$$

where $\omega(f, h)=\max \left\{\left|f\left(x_{1}\right)-f\left(x_{2}\right)\right|: x_{1}, x_{2} \in[a, b],\left|x_{1}-x_{2}\right| \leq h\right\}$.

For a solution $T_{n}$ of (1) let $A\left(T_{n}\right)=\left\{x \in[a, b]:\left|T_{n}(x)\right|=\left\|T_{n}\right\|_{C}\right\}$ and $Z\left(T_{n}\right)=\left\{x \in[a, b]: T_{n}(x)=0\right\}$ be the set of its extremal points and zeros, respectively. If $B \subset[a, b]$ is an arbitrary subset of $[a, b]$ then its distance from $[a, b]$ is measured by the quantity

$$
\rho(B)=\sup \{d-c:[c, d] \subset[a, b],[c, d] \cap B=\varnothing\} .
$$

For a function $g \in C[a, b]$ we say that it has $r$ sign changes in $[a, b]$ if there are points $a \leq x_{1}<\cdots<x_{r+1} \leq b$ such that $g\left(x_{i}\right) g\left(x_{i+1}\right)<0$, $1 \leq i \leq r$. (Recall, that a linear subspace $\mathscr{U}_{n} \subset C[a, b]$ is called a $W T$-space if its elements have at most $n-1$ sign changes in $[a, b]$.) Furthermore, $g$ has $r$ equioscillations if for some $a \leq x_{1}<\cdots<x_{r} \leq b$ we have $g\left(x_{i}\right)=$ $\xi(-1)^{i}\|g\|_{C}$, where $\xi=1$ or -1 and $1 \leq i \leq r$.

Theorem 1. Assume that $\mathscr{U}$ has finite codimension $r \in \mathbf{Z}_{+}$in $C[a, b]$ and suppose that for a given $n \in \mathbf{N}$ the elements of $\mathscr{U}_{n}=\operatorname{span}\left[\varphi_{1}, \ldots, \varphi_{n}\right]$ have at most $n+s_{n}-1$ sign changes $\left(s_{n} \in \mathbf{Z}_{+}\right)$. Then for any solution $T_{n}$ of $(1)$ having at least $n-k_{n}$ equioscillations $\left(0 \leq k_{n}<n\right) x_{1}, \ldots, x_{n-k_{n}}$ it holds that

$$
\max _{0 \leq i \leq n-k_{n}}\left(x_{i+1}-x_{i}\right) \leq 4(b-a)(r+1)\left(k_{n}+s_{n}+2\right) E\left(\mathscr{U} \cap W^{1}, \mathscr{U}_{n}\right)
$$

where $a=x_{0}, b=x_{n-k_{n}+1}$.

Corollary 1. Under the assumptions of Theorem 1 we have

$$
\begin{gathered}
\rho\left(A\left(T_{n}\right)\right) \leq 4(b-a)(r+1)\left(k_{n}+s_{n}+2\right) E\left(\mathscr{U} \cap W^{1}, \mathscr{U}_{n}\right), \\
\rho\left(Z\left(T_{n}\right)\right) \leq 8(b-a)(r+1)\left(k_{n}+s_{n}+2\right) E\left(\mathscr{U} \cap W^{1}, \mathscr{U}_{n}\right) .
\end{gathered}
$$

Remark. It is clear that $\mathscr{U}$ is a closed linear subspace of $C[a, b]$ and therefore by the Arzela-Ascoli theorem $\mathscr{U} \cap W^{1}$ is a compact set. Therefore

$$
\lim _{n \rightarrow \infty} E\left(\mathscr{U} \cap W^{1}, \mathscr{U}_{n}\right)=0 \text {. }
$$


Thus if $s_{n}$ and $k_{n}$ grow not faster than $1 / E\left(\mathscr{U} \cap W^{1}, \mathscr{U}_{n}\right)$ then (2) implies density of the extremal points and the zeros of general Chebyshev polynomials as $n \rightarrow \infty$. Theorem 1 holds for those general Chebyshev polynomials which have sufficiently large number of equioscillations. In our next theorem we replace this assumption by some sufficient conditions which ensure existence of general Chebyshev polynomials with "many" equioscillations.

Theorem 2. Let $\mathscr{U}$ have finite codimension $r \in \mathbf{Z}_{+}$in $C[a, b]$ and assume that for a given $n \in \mathbf{N}$ the space $\mathscr{U}_{n}=\operatorname{span}\left[\varphi_{1}, \ldots, \varphi_{n}\right]$ satisfies the following properties:

(i) the $\operatorname{span}\left[\varphi_{1}, \ldots, \varphi_{n-1}\right]$ contains an $\left(n-k_{n}-1\right)$-dimensional $W T$-subspace $\left(0 \leq k_{n}<n-1\right)$;

(ii) elements of $\mathscr{U}_{n}$ have at most $n+s_{n}-1$ sign changes $\left(s_{n} \geq 0\right)$.

Then there exists a solution $T_{n}$ of (1) with at least $n-k_{n}$ equioscillations for which (3) and (4) hold.

Moreover, if we replace (i) by the stronger assumption

$\left(\mathrm{i}^{\prime}\right)$ the $\operatorname{span}\left[\varphi_{1}, \ldots, \varphi_{n-1}\right]$ contains an $n-k_{n}-1$ dimensional $T$-subspace $\left(0 \leq k_{n}<n-1\right)$ then (3) and (4) hold for every solution $T_{n}$ of (1).

Let us consider a sequence of functions $\varphi_{1}, \varphi_{2}, \ldots, \varphi_{n}, \ldots$ in $C[a, b]$ and set $\mathscr{U}_{n}=\operatorname{span}\left[\varphi_{1}, \ldots, \varphi_{n}\right], n=1,2, \ldots$. Then our original problem (1) reduces to the special case when the spaces are imbedded, $\mathscr{U}_{n} \subset \mathscr{U}_{n+1}, n=$ $1,2, \ldots$, and $(1)$ is considered with respect to a certain basis. If we assume that $\mathscr{U}_{n}$ is a $W T$-space for every $n \in \mathbf{N}$ then conditions (i) and (ii) of Theorem 2 hold with $k_{n}=s_{n}=0$. Thus we obtain the following

Corollary 2. Let $\varphi_{1}, \ldots, \varphi_{n}, \ldots$ be a sequence of functions in $C[a, b]$ such that $\mathscr{U}_{n}=\operatorname{span}\left[\varphi_{1}, \ldots, \varphi_{n}\right]$ is a $W T$-space for every $n=1,2, \ldots$ and $\mathscr{U}$ has finite codimension $r \in \mathbf{Z}_{+}$in $C[a, b]$. Then there exists a solution $T_{n}$ of (1) $(n \in \mathbf{N})$ such that

$$
\rho\left(A\left(T_{n}\right)\right) \leq 8(b-a)(r+1) E\left(\mathscr{U} \cap W^{1}, \mathscr{U}_{n}\right)
$$

and

$$
\rho\left(Z\left(T_{n}\right)\right) \leq 16(b-a)(r+1) E\left(\mathscr{U} \cap W^{1}, \mathscr{U}_{n}\right) .
$$

If, in addition, the sequence $\left\{\varphi_{i}\right\}_{i=1}^{\infty}$ is closed in $C[a, b]($ i.e. $\mathscr{U}=C[a, b]$, $r=0)$ then for every $n \in \mathbf{N}$

$$
\begin{gathered}
\rho\left(A\left(T_{n}\right)\right) \leq 8(b-a) E\left(W^{1}, \mathscr{U}_{n}\right), \\
\rho\left(Z\left(T_{n}\right)\right) \leq 16(b-a) E\left(W^{1}, \mathscr{U}_{n}\right) .
\end{gathered}
$$

Note that if in the above corollary $\mathscr{U}_{n}$ 's are $T$-spaces then the general Chebyshev polynomial is unique for each $n \in \mathbf{N}$. In particular (6) and (7) hold for the general Chebyshev polynomials with respect to a closed Markov system $\left\{\varphi_{i}\right\}_{i=1}^{\infty}$.

Proof of Theorem 1. Let $T_{n}$ be a solution of (1) with equioscillations $T_{n}\left(x_{i}\right)=$ $\xi(-1)^{i}\left\|T_{n}\right\|_{C}$, where $\xi=1$ or -1 and $x_{i} \in[a, b], 1 \leq i \leq n-k_{n}$. Furthermore set $\tilde{T}_{n}=T_{n} /\left\|T_{n}\right\|_{C}$ and let $(\alpha, \beta) \subset[a, b]$ be such that $x_{i} \notin(\alpha, \beta)$, $1 \leq i \leq n-k_{n}$. Let $(\alpha, \beta)=\bigcup_{i=1}^{r+1}\left(\alpha_{i}, \beta_{i}\right)$ be the partition of $(\alpha, \beta)$ to $r+1$ equal intervals. Then we can construct piecewise linear functions $f_{i} \in C[a, b]$ 
such that $\left\|f_{i}\right\|_{C}=1, f_{i}(x)=0$ for $x \in[a, b] \backslash\left(\alpha_{i}, \beta_{i}\right), f_{i}$ equioscillates $k_{n}+s_{n}+2$ times and

$$
\omega\left(f_{i}, h\right) \leq \frac{2\left(k_{n}+s_{n}+2\right)}{\beta_{i}-\alpha_{i}} h=\frac{2(r+1)\left(k_{n}+s_{n}+2\right)}{\beta-\alpha} h
$$

for every $1 \leq i \leq r+1$. In addition we assume that the sign of the first oscillation of $f_{i}$ is opposite to the sign of the last oscillation of $T_{n}$ not exceeding $\alpha$ (if there is any), $1 \leq i \leq r+1$. Since functions $f_{1}, \ldots, f_{r+1}$ are linearly independent and codim $\mathscr{U}=r$ there exist $c_{1}, \ldots, c_{r+1}$ such that $\max _{1 \leq i \leq r+1}\left|c_{i}\right|=c_{j}=2(1 \leq j \leq r+1)$ and $f=\sum_{i=1}^{r+1} c_{i} f_{i} \in \mathscr{U}$. Consider the function $f^{*}=f+\tilde{T}_{n}$. Since $f=0$ on $[a, b] \backslash(\alpha, \beta)$ we have $f^{*}\left(x_{i}\right)=\tilde{T}_{n}\left(x_{i}\right)=\xi(-1)^{i}, 1 \leq i \leq n-k_{n}$. Furthermore, $f^{*}(x)=2 f_{j}(x)+\tilde{T}_{n}(x)$ for $x \in\left(\alpha_{j}, \beta_{j}\right)$, where $\left\|f_{j}\right\|_{C}=\left\|\tilde{T}_{n}\right\|_{C}=1$ and $f_{j}$ equioscillates $k_{n}+s_{n}+2$ times on $\left(\alpha_{j}, \beta_{j}\right)$ with the sign of its first oscillation being opposite to the sign of the last oscillation of $\tilde{T}_{n}$ not exceeding $\alpha$. Thus for some distinct points $y_{1}, \ldots, y_{m} \in[a, b], m=n+s_{n}+1$ we have

$$
\eta(-1)^{i} f^{*}\left(y_{i}\right) \geq 1 \quad\left(1 \leq i \leq n+s_{n}+1, \eta= \pm 1\right) .
$$

Now we claim that $E\left(f^{*}, \mathscr{U}_{n}\right) \geq 1$. Assume that to the contrary $\left\|f^{*}-p_{n}\right\|_{C}<1$ for some $p_{n} \in \mathscr{U}_{n}$. Then in view of (8) we have

$$
\eta(-1)^{i} p_{n}\left(y_{i}\right)>0, \quad 1 \leq i \leq n+s_{n}+1,
$$

i.e., $p_{n}$ has at least $n+s_{n}$ sign changes, contradicting the assumption of the theorem. Hence $E\left(f, \mathscr{U}_{n}\right)=E\left(f^{*}, \mathscr{U}_{n}\right) \geq 1$. On the other hand,

$$
\|f\|_{C} \leq \max _{1 \leq i \leq r+1}\left|c_{i}\right|=2, \quad \omega(f, h) \leq \frac{4(r+1)\left(k_{n}+s_{n}+2\right)}{\beta-\alpha} h .
$$

Therefore, setting $\tilde{f}=M f$, where

$$
M=\frac{\beta-\alpha}{4(b-a)(r+1)\left(k_{n}+s_{n}+2\right)}<\frac{1}{4}
$$

we obtain

$$
\|\tilde{f}\|_{C} \leq\|f\|_{C} / 4 \leq \frac{1}{2}, \quad \omega(\tilde{f}, h) \leq M \omega(f, h) \leq \frac{h}{b-a} .
$$

Thus $\tilde{f} \in W^{1} \cap \mathscr{U}$ and, evidently,

$$
E\left(W^{1} \cap \mathscr{U}, \mathscr{U}_{n}\right) \geq E\left(\tilde{f}, \mathscr{U}_{n}\right)=M E\left(f, \mathscr{U}_{n}\right) \geq M .
$$

Taking into account (9) this immediately implies (2).

Proof of Theorem 2. It is clear that Theorem 2 will follow from Theorem 1 by verifying that under conditon (i) (respectively, $\left.\left(i^{\prime}\right)\right)$ there exists a general Chebyshev polynomial (solution of (1)) having $n-k_{n}$ equioscillations (respectively, every general Chebyshev polynomial has $n-k_{n}$ equioscillations).

First, let us assume that (i) holds, i.e., $G_{n-1}=\operatorname{span}\left[\varphi_{1}, \ldots, \varphi_{n-1}\right]$ contains an $\left(n-k_{n}-1\right)$-dimensional $W T$-subspace $\left(0 \leq k_{n}<n-1\right)$. Then we can write $G_{n-1}=P \oplus M$, where $P$ is an $\left(n-k_{n}-1\right)$-dimensional $W T$-space and $M$ is a $k_{n}$-dimensional linear subspace of $G_{n-1}$. Consider now an arbitrary $f \in C[a, b]$ and let $g \in G_{n-1}, g=p+t \quad(p \in P, t \in M)$ be a best approximant 
of $f$ in $G_{n-1}$. Set $\bar{f}=f-t$. Obviously, $p$ is a best approximant of $\bar{f}$ in $P$. Now we shall use the following result of Jones-Karlovitz [5]: if $M_{k}$ is a $k$-dimensional $W T$-space, then for every $f \in C[a, b]$ there exist a best approximant $p^{*}$ of $f$ in $M_{k}$ such that $f-p^{*}$ equioscillates $k+1$ times. In view of this theorem $\bar{f}$ has a best approximant $\bar{p}$ in $P$ such that $\bar{f}-\bar{p}$ has $n-k_{n}$ equioscillations. Since both $p$ and $\bar{p}$ are best approximants of $\bar{f}$ in $P$ it follows that

$$
\|f-(\bar{p}+t)\|_{C}=\|\bar{f}-\bar{p}\|_{C}=\|\bar{f}-p\|_{C}=\|f-g\|_{C} .
$$

This yields that $\bar{p}+t \in G_{n-1}$, is also a best approximant of $f$ in $G_{n-1}$ where $f-(\bar{p}+t)=\bar{f}-\bar{p}$ has $n-k_{n}$ equioscillations. Therefore each $f \in C[a, b]$ has such a best approximant $g$ in $G_{n-1}$ for which $f-g$ has $n-k_{n}$ equioscillations. Setting, in particular, $f=\varphi_{n}$ we obtain the existence of a solution of (1) having $n-k_{n}$ equioscillations.

Assume now that $\left(\mathrm{i}^{\prime}\right)$ holds, and let $T_{n}$ be an arbitary solution of (1). Then (see $\left[15\right.$, p. 178]) there exist points $x_{1}, \ldots, x_{k}$ and numbers $a_{1}, \ldots, a_{k} \neq 0$ $(1 \leq k \leq n)$ such that $T_{n}\left(x_{i}\right)=\operatorname{sgn} a_{i}\left\|T_{n}\right\|_{C} \quad(1 \leq i \leq k)$ and $\sum_{i=1}^{k} a_{i} p\left(x_{i}\right)=0$ for every $p \in G_{n-1}=\operatorname{span}\left[\varphi_{1}, \ldots, \varphi_{n-1}\right]$. If there are less than $n-k_{n}-1$ sign changes in the set $\left[a_{1}, \ldots, a_{k}\right]$ then using that $G_{n-1}$ contains an $\left(n-k_{n}-1\right)$ dimensional $T$-subspace we can find a $p \in G_{n-1}$ for which $\operatorname{sgn} p\left(x_{i}\right)=a_{i}$ $(1 \leq i \leq k)$, a contradiction. Thus the set $\left[a_{1}, \ldots, a_{k}\right]$ contains at least $n-k_{n}-1$ sign changes i.e., $T_{n}$ has at least $n-k_{n}$ equioscillations.

\section{ApPlications}

Now we turn to different applications of Theorems 1 and 2.

Müntz polynomials. Let us consider first the easier case when $0<a<b$. Then for arbitary integers $0=m_{1}<\cdots<m_{n}<\cdots$ the space of Müntz polynomials $\mathscr{U}_{n}=\left\{x^{m_{1}}, \ldots, x^{m_{n}}\right\}$ satisfies the $T$-property on $[a, b]$. If we add the condition $\sum_{n=2}^{\infty} 1 / m_{n}=\infty$ then by the classical Müntz theorem $\mathscr{U}=$ $\mathrm{Cl}\left(\bigcup_{n=1}^{\infty} \mathscr{U}_{n}\right)=C[a, b]$ and Corollary 2 is applicable. Thus estimates (6) and (7) hold, that is extremal points and zeros of general Chebyshev polynomials are dense in $[a, b]$ as $n \rightarrow \infty$. Moreover, using Jackson-Müntz type theorems (see [11] for details) we can obtain some explicit estimates of the density.

Now we turn to the more difficult case of the interval $[-1,1]$. The difficulty here is caused by the fact that Müntz polynomials in general do not satisfy the $T$-property on $[-1,1]$. For an arbitrary sequence of integers $0=m_{1}<\cdots<$ $m_{n}<\cdots$ denote by $\gamma(m)$ the number of integers not exceeding $m$ which do not belong to the above sequence and set $\mathscr{U}_{n}=\operatorname{span}\left[x^{m_{1}}, \ldots, x^{m_{n}}\right], n=$ $1,2, \ldots$. It can be easily seen that if the Müntz condition (sum of reciprocals is infinite) is fulfilled by both even and odd integers in the above sequence then $\mathrm{Cl}\left(\bigcup_{n=1}^{\infty} \mathscr{U}_{n}\right)=C[-1,1]$. Moreover, it is known (see [12]) that in order for $\mathscr{U}_{n}$ to satisfy the $T$-property on $[-1,1]$ it is necessary and sufficient, that $m_{i+1}-m_{i}$ is odd for every $1 \leq i \leq n-1$. Since $\mathscr{U}_{n}$ contains at most $\gamma\left(m_{n}\right)$ "gaps" this result implies that $\mathscr{U}_{n-1}$ has an $\left(n-\gamma\left(m_{n}\right)-1\right)$-dimensional $T$-subspace and $\mathscr{U}_{n}$ belongs to a $n+\gamma\left(m_{n}\right)$-dimensional $T$-subspace (see [6, p. 386] for details), if $\gamma\left(m_{n}\right)<n-1$. Thus we can apply Theorem 2 with $s_{n}=k_{n}=\gamma\left(m_{n}\right)$ and 
$r=0$, and hence for every solution $T_{n}$ of the extremal problem

$$
\min _{a_{i}}\left\|x^{m_{n}}-\sum_{i=1}^{n-1} a_{i} x^{m_{i}}\right\|_{C}
$$

it holds that

$$
\begin{aligned}
& \rho\left(A\left(T_{n}\right)\right) \leq 8\left(2 \gamma\left(m_{n}\right)+2\right) E\left(W^{1}, \mathscr{U}_{n}\right), \\
& \rho\left(Z\left(T_{n}\right)\right) \leq 16\left(2 \gamma\left(m_{n}\right)+2\right) E\left(W^{1}, \mathscr{U}_{n}\right) .
\end{aligned}
$$

Now restricting in suitable way the rate of growth of $\gamma(m)$ (as $m \rightarrow \infty)$ we can achieve that $E\left(W^{1}, \mathscr{U}_{n}\right)=o\left(1 / \gamma\left(m_{n}\right)\right)$ (here we can use again Jackson-Müntz type theorems). This leads to the density of extremal points and zeros of every solution of (10) as $n \rightarrow \infty$.

Restricted approximation. Consider a sequence of functions $\varphi_{1}, \varphi_{2}, \ldots, \varphi_{n}$, $\ldots$ in $C[a, b]$ such that $\mathscr{U}_{n}=\operatorname{span}\left[\varphi_{1}, \ldots, \varphi_{n}\right]$ is a $T$-space for every $n \geq 1$ and $\mathrm{Cl}\left(\bigcup_{n=1}^{\infty} \mathscr{U}_{n}\right)=C[a, b]$. Let $L_{1}, \ldots, L_{r}$ be bounded linear functionals from $C[a, b]$ to $\mathbf{R}$ which are linearly independent on $\mathscr{U}_{r}$. Set $\mathscr{U}_{n}^{*}=\{p \in$ $\left.\mathscr{U}_{n+r}: L_{i}(p)=0,1 \leq i \leq r\right\}, n=1,2, \ldots$. Since $\mathscr{U}_{n+1}^{*} \supset \mathscr{U}_{n}^{*}(n \geq 1)$ there exists a sequence of functions $\varphi_{1}^{*}, \ldots, \varphi_{n}^{*}, \ldots$ in $C[a, b]$ such that $\mathscr{U}_{n}^{*}=$ $\operatorname{span}\left[\varphi_{1}^{*}, \ldots, \varphi_{n}^{*}\right]$. Now consider the extremal problem

$$
\min _{a_{i}}\left\|\varphi_{n}^{*}-\sum_{i=1}^{n-1} a_{i} \varphi_{i}^{*}\right\|_{C}
$$

It is an easy exercise to verify that

$$
E\left(f, \mathscr{U}_{n}^{*}\right) \leq K_{r} E\left(f, \mathscr{U}_{n+r}\right)
$$

for every $f \in G=\left\{g \in C[a, b]: L_{i}(g)=0,1 \leq i \leq r\right\}$, where $K_{r}>0$ is independent of $f$ and $n$. In particular, $\operatorname{Cl}\left(\bigcup_{n=1}^{\infty} \mathscr{U}_{n}^{*}\right)=G$ and codim $G=r$ (in $C[a, b]$ ). Furthermore, the elements of $\mathscr{U}_{n}^{*}$ have at most $n+r-1$ zeros. Thus using Theorem 1 with $s_{n}=r$ we obtain by (12) that for every solution $T_{n}$ of (11) with $n-k_{n}$ equioscillations

$$
\begin{aligned}
\rho\left(A\left(T_{n}\right)\right) & \leq 4(b-a)(r+1)\left(k_{n}+r+2\right) E\left(G \cap W^{1}, \mathscr{U}_{n}^{*}\right) \\
& \leq 4 K_{r}(b-a)(r+1)\left(k_{n}+r+2\right) E\left(W^{1}, \mathscr{U}_{n+r}\right) .
\end{aligned}
$$

For example, if $L_{i}(f)=f\left(x_{i}\right), 1 \leq i \leq r$, for some $x_{1}, \ldots, x_{r} \in[a, b]$ then any solution $T_{n}$ of (11) should have at least $n-r$ equioscillations. Indeed, there exists a $p \in \mathscr{U}_{n-1}^{*}$ having nonnodal zeros at $x_{1}, \ldots, x_{r}$ (zeros without sign change), changing its sign at prescribed points $y_{1}, \ldots, y_{k}, 1 \leq k \leq n-r-2$ $\left(y_{i} \neq x_{j}\right)$, and having no other zeros in $(a, b)$ (see [4, p. 30]). This and the argument used in the proof of Theorem 2 imply that any solution of (11) has at least $n-r$ equioscillations. Hence $k_{n}=r$ in this case and by (13)

$$
\rho\left(A\left(T_{n}\right)\right) \leq 8 K_{r}(b-a)(r+1)^{2} E\left(W^{1}, \mathscr{U}_{n+r}\right) .
$$

Distribution of zeros and extremal points of error functions. Let $f \in C[a, b]$ and consider a sequence of linear subspaces $G_{n}(n=1,2, \ldots)$ in $C[a, b]$. If $p_{n}(f) \in G_{n}$ is a best approximant of $f$ in $G_{n}(n=1,2, \ldots)$ then an interesting question consists in studying the distribution of zeros and extremal points of the error functions $R_{n}(f)=f-p_{n}(f)$ as $n \rightarrow \infty$. (In what follows we 
shall discuss only the distribution of extremal points, all statements hold true for zeros, as well). For the case $G_{n}=P_{n}=\operatorname{span}\left[1, x, \ldots, x^{n-1}\right]$ this question was studied in [3 and 16]. Kadec [3] showed that for every $f \in C[-1,1]$ (which is not a polynomial) and $[\alpha, \beta] \subset[-1,1]$ :

$$
\limsup _{n \rightarrow \infty} \frac{A_{n}(f,[\alpha, \beta])}{n} \geq \frac{\arccos \alpha-\arccos \beta}{\pi}
$$

where $A_{n}(f,[\alpha, \beta])$ denotes the number of extremal points of $R_{n}(f)$ lying in $[\alpha, \beta]$. Tashev [16] proved that for every $f \in C[-1,1]$

$$
\liminf _{n \rightarrow \infty} \frac{n}{\ln n} \rho\left(A\left(R_{n}(f)\right)\right)<\infty
$$

Thus by (14) and (15) for every $f \in C[a, b]$ there is a "good" subsequence of $n$ 's for which extremal points of $R_{n}(f)$ are nicely distributed. Lorentz [10] verified that the above statements can not hold for every $n$. He constructed an entire function $f$ for which $A\left(R_{n}(f)\right) \cap[0,1]=\varnothing$ for infinitely many $n$ 's. (The extremal problem being considered on $[-1,1]$.) This raises the natural question (first posed by Lorentz [10]) for what classes of functions the results of Kadec and Tashev hold for every $n$.

We shall use our results for giving a certain answer to the above question. For a sequence of linear subspaces $G_{n}=\operatorname{span}\left[g_{1}, \ldots, g_{n}\right] \subset C[a, b] \quad(n=$ $1,2, \ldots)$ and a given $f \in C[a, b]$ set $\mathscr{U}_{n}=\operatorname{span}\left[G_{n-1}, f\right]$ and consider the extremal problem

$$
\min _{a_{i}}\left\|f-\sum_{i=1}^{n-1} a_{i} g_{i}\right\|_{C} .
$$

In order to apply our results we need the following quantity

$$
\tau\left(f, G_{n-1}\right)=\sup _{p \in G_{n-1}}\{\text { number of sign changes of } f+p\}-n+1 .
$$

(Evidently, $\tau\left(f, G_{n-1}\right) \geq 0$ for every $f$.) Any solution $T_{n}$ of (16) can be written as $T_{n}=f-p_{n-1}^{*}(f)=R_{n-1}(f)$, where $p_{n-1}^{*}(f)$ is a best approximant of $f$ in $G_{n-1}$.

If we assume now that $G_{n}(n=1,2, \ldots)$ are $W T$-spaces and $\mathrm{Cl}\left(\bigcup_{n=1}^{\infty} G_{n}\right)$ $=C[a, b]$ then applying Theorem 2 with $r=0, k_{n}=0, s_{n}=\tau\left(f, G_{n-1}\right)$ and $\mathscr{U}_{n}=\operatorname{span}\left[g_{1}, \ldots, g_{n-1}, f\right]$ we obtain

Theorem 3. Let $G_{n}=\operatorname{span}\left[g_{1}, \ldots, g_{n}\right], n=1,2, \ldots$, be a sequence of $W T$ spaces in $C[a, b]$ such that $\mathrm{Cl}\left(\bigcup_{n=1}^{\infty} G_{n}\right)=C[a, b]$ and consider an arbitrary $f \in C[a, b]$. Then for every $n \in \mathbf{N}$ there exists a best approximant $p_{n-1}^{*}(f)$ of $f$ in $G_{n-1}$ such that

$$
\rho\left(A\left(R_{n-1}(f)\right)\right) \leq 4(b-a)\left(\tau\left(f, G_{n-1}\right)+2\right) E\left(W^{1}, G_{n-1}\right),
$$

where $R_{n-1}(f)=f-p_{n-1}^{*}(f)$.

Corollary 3. Let $G_{n}=\operatorname{span}\left[g_{1}, \ldots, g_{n}\right], n=1,2, \ldots$, be a sequence of $T$ spaces in $C[a, b]$ such that

$$
E\left(W^{1}, G_{n}\right)=O(1 / n) .
$$


Then for every $f \in C[a, b]$ and $n \in \mathbf{N}$ we have

$$
\rho\left(A\left(R_{n}(f)\right)\right)=O\left(\frac{\tau\left(f, G_{n}\right)}{n}\right) .
$$

Thus by the above corollary we can ensure that $\lim _{n \rightarrow \infty} \rho\left(A\left(R_{n}(f)\right)\right)=0$ if $\tau\left(f, G_{n}\right)=o(n)$. Moreover, if $\tau\left(f, G_{n}\right)=O(1)$ then $\rho\left(A\left(R_{n}(f)\right)\right)=O(1 / n)$. This presents a certain class of functions, for which Tashev's result can be extended for every $n$.

Example 1. If $f$ is a rational function or a trigonometric polynomial, then $\tau\left(f, P_{n}\right)=O(1)$.

Example 2. If $f \in C^{\infty}[a, b]$ is a generalized convex function, i.e. $f^{(k)} \geq 0$ (or $\leq 0), k=1,2, \ldots$, then $\tau\left(f, P_{n}\right)=0$. (In the special case, when $\tau\left(f, P_{n}\right)=$ 0 the density of extremal points of $R_{n}(f)$ as $n \rightarrow \infty$ also follows from an "interlacing" type result of Pinkus and Ziegler [13].)

By Corollary $3 \lim _{n \rightarrow \infty} \rho\left(A\left(R_{n}(f)\right)\right)=0$ if $\tau\left(f, G_{n}\right)=o(n)$. For the special case when $G_{n}=P_{n}$ we can even prove the uniform distribution (with respect to Chebyshev measure) of extremal points of $R_{n}(f)$ for every $n$ if the condition $\tau\left(f, G_{n}\right)=o(n)$ holds, i.e., under this latter condition Kadec's theorem is valid for every $n$.

Theorem 4. If for a given $f \in C[-1,1]$ which is not a polynomial $\tau\left(f, P_{n}\right)=$ $o(n)$ then

$$
\lim _{n \rightarrow \infty} \frac{A_{n}(f,[\alpha, \beta])}{n} \geq \frac{\arccos \alpha-\arccos \beta}{\pi}
$$

for every $[\alpha, \beta] \subset[-1,1]$.

Proof. Let $p_{n}^{*}(f)$ be the best approximant of $f$ in $P_{n}$ and let $x_{1}, \ldots, x_{n+1} \in$ $[-1,1]$ be such that

$$
f\left(x_{i}\right)-p_{n}^{*}\left(f, x_{i}\right)=\xi(-1)^{i} E\left(f, P_{n}\right) \quad(\xi= \pm 1,1 \leq i \leq n+1) .
$$

Choosing an integer $s \in \mathbf{N}$ satisfying $\sqrt{n}<s<2 \sqrt{n}$ we consider the function

$$
g(x)=\frac{f(x)-p_{n}^{*}(f, x)}{E\left(f, P_{n}\right)}+\frac{1}{2}(x+1-b)^{s} \cos (n-s) \arccos x,
$$

where $-1<b<1$. Since $|x+1-b| \leq 1$ for $x \in[-1, b]$ it follows that $\xi(-1)^{i} g\left(x_{i}\right) \geq \frac{1}{2}$ for $x_{i} \in[-1, b]$. This means that $g$ has at least $\tilde{A}_{n}(f,[-1, b])-1$ sign changes in $[-1, b]$ where $\tilde{A}_{n}(f,[\alpha, \beta])$ denotes the number of $x_{i}$ 's, $1 \leq i \leq n+1$, belonging to $[\alpha, \beta] \subset[-1,1]$. Moreover, for every $\bar{x} \in[b+1 / \sqrt{n}, 1]$ such that $|\cos (n-s) \arccos \bar{x}|=1$

$$
\left|\frac{1}{2}(\bar{x}+1-b)^{s} \cos (n-s) \arccos \bar{x}\right| \geq \frac{1}{2}\left(1+\frac{1}{\sqrt{n}}\right)^{s}>\frac{1}{2}\left(1+\frac{1}{\sqrt{n}}\right)^{\sqrt{n}}>1 \text {. }
$$

Thus $g$ has at least

$$
\frac{\arccos (b+1 / \sqrt{n})}{\pi}(n-s)+o(1) \geq \frac{n \arccos b}{\pi}+o(n)
$$

sign changes in $(b, 1]$. Therefore in total $g$ should have at least

$$
\tilde{A}_{n}(f,[-1, b])+\frac{n \arccos b}{\pi}+o(n)
$$


sign changes in $[-1,1]$. On the other hand $g(x)=c\left(f(x)+p_{n}(x)\right)$ for some $c \in \mathbf{R}$ and $p_{n} \in P_{n}$, i.e., by the assumption of the theorem $g$ may have at most $\tau\left(f, P_{n}\right)+n=n+o(n)$ sign changes. This yields that

$$
\tilde{A}_{n}(f,[-1, b])+\frac{n \arccos b}{\pi} \leq n+o(n)
$$

that is

$$
\frac{\tilde{A}_{n}(f,[-1, b])}{n} \leq \frac{\arccos (-1)-\arccos b}{\pi}+o(1) .
$$

Analogously,

$$
\frac{\tilde{A}_{n}(f,[b, 1])}{n} \leq \frac{\arccos b}{\pi}+o(1) .
$$

Combining (17) with $\tilde{A}_{n}(f,[-1,1]) \geq n+1$ we have

$$
\frac{\tilde{A}_{n}(f,[b, 1])}{n} \geq \frac{\arccos b}{\pi}+o(1) \text {. }
$$

Thus and by (18)

$$
\tilde{A}_{n}(f,[b, 1])=n \frac{\arccos b}{\pi}+o(n)
$$

for every $-1<b<1$. Analogously,

$$
\tilde{A}_{n}(f,[-1, b])=n \frac{\arccos (-1)-\arccos b}{\pi}+o(n)
$$

for every $-1<b<1$. This completes the proof of the theorem.

Let us conclude this part of the paper by remarking that all results presented above with minor modifications are also valid for appoximating on finite union of disjoint intervals.

\section{COUNTEREXAMPLES}

Now we turn to the discussion of the necessity of different assumptions used in order to obtain positive results. Theorems 1 and 2 provided some estimates for the density of extremal points and zeros of general Chebyshev polynomials (with respect to $\mathscr{U}_{n}=\operatorname{span}\left[\varphi_{1}, \ldots, \varphi_{n}\right]$ ) under the following condition:

(a) the limit of $\mathscr{U}_{n}$ 's has finite codimension in $C[a, b]$;

(b) general Chebyshev polynomials have a sufficiently large number of equioscillations;

(c) elements of $\mathscr{U}_{n}$ have a limited number of sign changes.

We shall verify in this section that all three conditions are in general necessary for the density of extremal points and zeros.

Most of our counterexamples are based on the so-called incomplete polynomials. Following [14] we call a polynomial $p(x)=\sum_{i=0}^{n} a_{i} x^{i} \theta$-incomplete $(0<\theta<1)$ if $a_{i}=0$ for $0 \leq i \leq s-1$ and $s / n \geq \theta$. It is known [14, p. 226] that if $p$ is $\theta$-incomplete and $|p(\xi)|=\max _{x \in[0,1]}|p(x)| \neq 0$, then $\xi \geq \theta^{2}$. Moreover, a uniformly bounded sequence of $\theta$-incomplete polynomials of growing degrees tends to 0 for every $x \in\left[0, \theta^{2}\right)$. 
Example A. Set for $n \in \mathbf{N}$

$$
\mathscr{U}_{n}=\operatorname{span}\left[x^{\theta_{n}}, \ldots, x^{n+\theta_{n}-1}\right] \quad(x \in[0,1])
$$

where $\theta_{n} \in \mathbf{N}$ and $\theta_{n} \geq \frac{\theta}{1-\theta}(n-1), 0<\theta<1$. Then elements of $\mathscr{U}_{n}$ are $\theta$-incomplete polynomials. Evidently, $\mathscr{U}$ has infinite codimension in this case and the unique solution of

$$
\min _{a_{i}}\left\|x^{n+\theta_{n}-1}-\sum_{i=\theta_{n}}^{n+\theta_{n}-2} a_{i} x^{i}\right\|_{C}
$$

has no extremal points in $\left[0, \theta^{2}\right)$. This shows that density may fail in the case when $\mathscr{U}_{n}$ 's are $W T$-spaces $\left(k_{n}=s_{n}=0\right)$, but $\mathscr{U}$ has infinite codimension.

Let now the sequence of subspaces $\mathscr{U}_{n}, n=1,2, \ldots$, in $C[a, b]$ satisfy relations

$$
E\left(W^{1}, \mathscr{U}_{n}\right) \leq K / n,
$$

where $K>0$ is a given constant. In addition, we also assume that elements of $\mathscr{U}_{n}$ have at most $n+s_{n}-1$ sign changes in $[a, b]$. Then by Theorem 1 for any solution $T_{n}$ of (1) with at least $n-k_{n}$ equioscillations we have

$$
\rho\left(A\left(T_{n}\right)\right) \leq 4 K(b-a) \frac{k_{n}+s_{n}+2}{n} .
$$

Hence, in particular, the conditions

$$
k_{n}=o(n), \quad s_{n}=o(n)
$$

yield density of extremal points as $n \rightarrow \infty$. This raises the natural question if we can get density under weaker assumptions on $k_{n}$ and $s_{n}$. Our next two examples show that density may fail if at least one of the conditions (21) does not hold.

Example B. We shall construct subspaces $\mathscr{U}_{n}, n=1,2, \ldots$, in $C[0,1]$ such that (20) holds, $s_{n}=0$ (i.e. elements of $\mathscr{U}_{n}$ have at most $n-1$ sign changes) and density fails for a sequence of general Chebyshev polynomials having $n-k_{n}$ equioscillations with $k_{n} \geq n \theta, 0<\theta<1 / 2$ (i.e. condition $k_{n}=o(n)$ does not hold). Let $T_{n}^{*}$ be the solution of

$$
\min _{a_{i}}\left\|x^{n-1}-\sum_{i=k_{n}}^{n-2} a_{i} x^{i}\right\|_{C}, \quad n \theta \leq k_{n} \leq \frac{n}{2}, 0<\theta<\frac{1}{2} .
$$

Then $T_{n}^{*}$ is uniquely defined and has $n-k_{n}$ equioscillations $x_{1}, \ldots, x_{n-k_{n}}$ which do not belong to $\left[0, \theta^{2}\right)$ (since $T_{n}^{*}$ is $\theta$-incomplete). Let $G_{k_{n}}=\{p \in$ $\left.P_{n}: p\left(x_{i}\right)=0,1 \leq i \leq n-k_{n}\right\}=\operatorname{span}\left[\tilde{\varphi}_{i}, n-1 \geq i \geq n-k_{n}\right]$ and set $\mathscr{U}_{n}=$ $\operatorname{span}\left[\varphi_{1}, \ldots, \varphi_{n}\right]$, where $\varphi_{i}(x)=x^{i-1}, 1 \leq i \leq n-k_{n}-1 ; \varphi_{i}(x)=\tilde{\varphi}_{i}(x)$, $n-k_{n} \leq i \leq n-1, \varphi_{n}(x)=T_{n}^{*}(x)$. Evidently, $\mathscr{U}_{n}=P_{n}$, i.e. our construction consists in just choosing a special basis in $P_{n}$. In particular, $s_{n}=0$ and (20) holds. Now we claim that

$$
\min _{a_{i}}\left\|\varphi_{n}-\sum_{i=1}^{n-1} a_{i} \varphi_{i}\right\|_{C}=\left\|\varphi_{n}\right\|_{C}=\left\|T_{n}^{*}\right\|_{C} .
$$


That is $T_{n}^{*}$ is a general Chebyshev polynomial with respect to the above basis in $P_{n}$. Assume that

$$
\left\|T_{n}^{*}-p\right\|_{C}<\left\|T_{n}^{*}\right\|_{C}
$$

for some $p=\sum_{i=1}^{n-1} a_{i} \varphi_{i}=p_{1}+p_{2}$, where $p_{1} \in P_{n-k_{n}-1}$ and $p_{2} \in G_{k_{n}}$. Since $T_{n}^{*}\left(x_{i}\right)=\xi(-1)^{i}\left\|T_{n}^{*}\right\|_{C}, \xi= \pm 1,1 \leq i \leq n-k_{n}$, it follows from (22) that $\xi(-1)^{i} p\left(x_{i}\right)>0$ for every $1 \leq i \leq n-k_{n}$. But $p_{2}\left(x_{i}\right)=0,1 \leq i \leq n-k_{n}$, hence $\xi(-1)^{i} p_{1}\left(x_{i}\right)>0,1 \leq i \leq n-k_{n}$. This means that $p_{1} \in P_{n-k_{n}-1}$ has at least $n-k_{n}-1$ zeros, i.e., $p_{1} \equiv 0$. Thus $p\left(x_{i}\right)=p_{2}\left(x_{i}\right)=0 \quad\left(1 \leq i \leq n-k_{n}\right)$, contradicting (22). Therefore $T_{n}^{*}$ is a general Chebyshev polynomial having $n-k_{n}$ equioscillations not belonging to $\left[0, \theta^{2}\right)$.

Example C. Finally, we construct subspaces $\mathscr{U}_{n}, n=1,2, \ldots$, in $C[0,1]$ such that (20) holds and general Chebyshev polynomials have $n$ equioscillations (i.e. $k_{n}=0$ ), but density fails because elements of $\mathscr{U}_{n}$ may have $n+s_{n}-1$ sign changes with $s_{n} \geq \alpha n, 0<\alpha<1$ (condition $s_{n}=o(n)$ does not hold). Let $\tilde{T}_{n}$ be the solution of (19) $\left(\theta_{n} \geq \frac{\theta}{1-\theta}(n-1)\right)$ and set $\mathscr{U}_{n}=\operatorname{span}\left[1, x, \ldots, x^{n-2}, \tilde{T}_{n}\right]$. Since $\tilde{T}_{n}$ has $n$ equioscillations it is also the general Chebyshev polynomial for $\mathscr{U}_{n}$ with the above basis. Thus (20) holds and $k_{n}=0$. On the other hand $\tilde{T}_{n}$ is a $\theta$-incomplete polynomial and therefore density of extremal points fails. This failure is due to the fact that $s_{n}=\theta_{n} \geq \frac{\theta}{1-\theta}(n-1)$, that is $s_{n} \neq o(n)$.

Remark. It can be easily seen that all above examples of nondensity of extremal points also hold with respect to the zeros of general Chebyshev polynomials.

It is more difficult to obtain counterexamples in the case when we have an infinite Markov system of functions $\varphi_{1}, \varphi_{2}, \ldots, \varphi_{n}, \ldots$ in $C[a, b]$, that is $\mathscr{U}_{n}=\operatorname{span}\left[\varphi_{1}, \ldots, \varphi_{n}\right]$ is a $T$-space for every $n=1,2, \ldots$, and we consider extremal problem (1) with respect to the basis $\left[\varphi_{1}, \ldots, \varphi_{n}\right], n=1,2, \ldots$.

We want to show now that even in the case of an infinite Markov system denisty of extremal points (zeros) of general Chebyshev polynomials may fail if the closure of the system has infinite codimension. (Here we have, of course, $k_{n}=s_{n}=0$.) We shall give our statement in a more general form of an $L_{p^{-}}$ extremal problem $(1 \leq p \leq \infty)$.

For given numbers $\alpha_{j} \in \mathbf{R} \backslash[-1,1], j=1,2, \ldots$, set $\mathscr{U}_{n+1}=R\left(\alpha_{1}, \ldots, \alpha_{n}\right)$ $=\operatorname{span}\left[1,1 /\left(x-\alpha_{1}\right), \ldots, 1 /\left(x-\alpha_{n}\right)\right]$ and denote by $r_{n, p}\left(\alpha_{1}, \ldots, \alpha_{n}\right)$ the corresponding general $L_{p}$-Chebyshev polynomial:

$$
\begin{aligned}
\left\|r_{n, p}\left(\alpha_{1}, \ldots, \alpha_{n}\right)\right\|_{p} \\
=\min _{a_{i}}\left\|\frac{1}{x-\alpha_{n}}-\left(\sum_{j=1}^{n-1} \frac{a_{j}}{x-\alpha_{j}}+a_{0}\right)\right\|_{p} \quad(1 \leq p \leq \infty) .
\end{aligned}
$$

(We consider here the $L_{p}$-norm on $[-1,1]$ if $1 \leq p<\infty$, for $p=\infty$ the norm in (23) is the supremum norm on $[-1,1]$.)

Theorem 5. For any $-1<c<-1 / 2$ there exists a sequence $1<\alpha_{j}<1+1 / j^{4}$, $j=1,2, \ldots$, such that $r_{n, p}\left(x, \alpha_{1}, \ldots, \alpha_{n}\right)$ has no zeros in $[-1, c]$ for every $n \in \mathbf{N}$ and $1 \leq p \leq \infty$.

In the case when $p=\infty$ the above statement was proved (by a different method) in [2]. 
We shall need some additional notations. For the sequence $\alpha_{j} \in \mathbf{R} \backslash[-1,1]$, $j=1,2, \ldots$, set

$$
q_{n}(x)=\prod_{j=1}^{n}\left(\alpha_{j}-x\right), \quad q_{n} \in P_{n+1}
$$

Obviously,

$$
R\left(\alpha_{1}, \ldots, \alpha_{n}\right)=\left\{\frac{p(x)}{q_{n}(x)}: p \in P_{n+1}\right\}
$$

is an $(n+1)$-dimensional $T$-space on $[-1,1]$. Furthermore, for $t_{j} \in(-1,1)$ defined by $\left(1+t_{j}^{2}\right) / 2 t_{j}=\alpha_{j} \quad(j \in \mathbf{N})$ set $g_{n}(z)=\prod_{j=1}^{n}\left(z-t_{j}\right)$. Then

$$
q_{n}(\cos \varphi)=c\left|g_{n}\left(e^{i \varphi}\right)\right|^{2}, \quad \varphi \in[0, \pi],
$$

where $c \in \mathbf{R} \backslash\{0\}$. In what follows $q_{n}$ and $g_{n}$ will always denote polynomials defined by $\alpha_{j}$ 's as above. Let us note that it follows from [1, p. 255] that if $1-t_{j}=O\left(j^{-\beta}\right)$ with some $\beta>1$ (i.e. $\left.\alpha_{j}-1=O\left(j^{-2 \beta}\right)\right)$ then $\mathrm{Cl}\left(\bigcup_{n=1}^{\infty} R\left(\alpha_{1}, \ldots, \alpha_{n}\right)\right)$ has infinite codimension in $C[-1,1]$, Finally, set

$$
\tilde{S}_{n}(x)=\tilde{S}_{n}\left(x, \alpha_{1}, \ldots, \alpha_{n-1}\right)=\operatorname{Im}\left\{z^{-(2 n-5) / 2} g_{n-1}^{2}(z)\right\} / \sin \frac{\varphi}{2},
$$

where $z=e^{i \varphi}, x=\cos \varphi$. It is easy to verify that $\tilde{S}_{n}$ is a polynomial of degree $n$. It follows from [1, p. 253] that

$$
\int_{-1}^{1} x^{j}\left|\tilde{S}_{n}(x)\right|^{p-1} \operatorname{sgn} \tilde{S}_{n}(x) \tilde{w}(x) d x=0 \quad(1 \leq p<\infty, 0 \leq j \leq n-1)
$$

where $\tilde{w}(x)=(1-x)^{(p-1) / 2} /(1+x)^{1 / 2} q_{n-1}^{p}$.

Proof of Theorem 5. First we claim that there exists a sequence of numbers $1<\alpha_{j}<1+1 / j^{4} \quad(j \in \mathbf{N})$ such that $\tilde{S}_{n}(x)$ has no zeros in $[-1, c](c \in$ $(-1,-1 / 2))$. We just give a sketch of the proof of this claim since it is similar to the proof of Lemma 3 in [8]. Set $\alpha=\arccos c \in(2 \pi / 3, \pi)$ and let $\varepsilon_{j} \in \mathbf{R}_{+}$, $j=1,2, \ldots$, satisfy

$$
\sum_{j=1}^{\infty} \varepsilon_{j}<\frac{3}{2} \alpha-\pi
$$

Now we can choose $t_{j} \in\left(1-\frac{1}{2} j^{-2}, 1\right)$ such that

$$
\pi-\varepsilon_{j} \leq \arg \left(\frac{e^{i \alpha}-t_{j}}{1-t_{j} e^{i \alpha}}\right) \quad(j \in \mathbf{N}) .
$$

Setting $g_{n-1}(z)=\prod_{j=1}^{n-1}\left(z-t_{j}\right), g_{n-1}^{*}(z)=z^{n} g_{n-1}(1 / z)$, and using (28), (29) and the fact that $\arg \left[g_{n-1}(z) / g_{n-1}^{*}(z)\right]$ is monotone increasing in $\varphi(\varphi=\arg z)$ we get for $\varphi \in[\alpha, \pi)$

$$
\arg z^{3 / 2}+\arg \frac{g_{n-1}(z)}{g_{n-1}^{*}(z)} \geq \frac{3}{2} \alpha+\sum_{j=1}^{n-1}\left(\pi-\varepsilon_{j}\right)>n \pi .
$$

Since $\varphi_{0}$ is a zero of $\operatorname{Im}\left\{e^{-i \varphi(2 n-5) / 2} g_{n-1}^{2}\left(e^{i \varphi}\right)\right\}$ if and only if

$$
\arg e^{3 i \varphi_{0} / 2}+\arg \frac{g_{n-1}\left(e^{i \varphi_{0}}\right)}{g_{n-1}^{*}\left(e^{i \varphi_{0}}\right)}=k \pi, \quad k=1,2, \ldots,
$$


it follows from (30) that $\tilde{S}_{n}(x)=\tilde{S}_{n}(\cos \varphi)$ has all zeros in $(0, \alpha)$. Since $\alpha_{j}=\left(1+t_{j}^{2}\right) / 2 t_{j}$ the relations $1-1 / 2 j^{2}<t_{j}<1$ imply $\alpha_{j}<1+1 / j^{4}$.

Thus we have verified our claim.

We can represent the solution of (23) as

$$
r_{n, p}\left(x, \alpha_{1}, \ldots, \alpha_{n}\right)=\frac{p_{n, p}^{*}(x)}{q_{n}(x)}
$$

where $p_{n, p}^{*}(x) \in P_{n+1}$. Using the well-known orthogonality property of best $L_{p}$-approximants we have

$$
\int_{-1}^{1} \frac{x^{j}}{q_{n-1}(x)}\left|\frac{p_{n, p}^{*}(x)}{q_{n}(x)}\right|^{p-1} \operatorname{sgn} p_{n, p}^{*}(x) d x=0, \quad 0 \leq j \leq n-1 .
$$

Since $q_{n}(x)=\left(\alpha_{n}-x\right) q_{n-1}(x)$ it follows that

$$
\begin{aligned}
& \int_{-1}^{1} \frac{x^{j}}{q_{n-1}^{p}(x)\left(\alpha_{n}-x\right)^{p-1}}\left|p_{n, p}^{*}(x)\right|^{p-1} \operatorname{sgn} p_{n, p}^{*}(x) d x=0, \\
& \quad 0 \leq j \leq n-1 .
\end{aligned}
$$

Thus writing $p_{n, p}^{*}(x)=a_{n} x^{n}+\cdots$ we have

$$
\left\|p_{n, p}^{*} w\right\|_{p}=\min _{a_{0}, \ldots, a_{n-1}}\left\|\left(a_{n} x^{n}+a_{n-1} x^{n-1}+\cdots+a_{0}\right) w\right\|_{p}
$$

where $w(x)=1 / q_{n-1}^{p}(x)\left(\alpha_{n}-x\right)^{p-1}$. Set $\bar{w}(\tau, x)=(1-\tau) \tilde{w}(x)+\tau w(x)$, where $\tilde{w}(x)=(1-x)^{(p-1) / 2} /(1+x)^{1 / 2} q_{n-1}^{p}$, and (27) holds with this weight. Obviously $\frac{\partial \bar{w}}{\partial \tau} / \bar{w}$ is an increasing function of $x \in(-1,1)$ for every $0 \leq \tau<1$ (since $\frac{d}{d x} \frac{w}{\tilde{w}}>0$ ) and therefore by Theorem 1 of [9] the zeros of the polynomials of minimal $L_{p}$-norm with respect to the weight $\bar{w}(\tau, x)$ are increasing functions of $\tau$. Thus using (27) and (31) we obtain that $j$ 's zero of $\tilde{S}_{n}(x)$ is smaller, than $j$ 's zero of $p_{n, p}^{*}(x)$. Since $\tilde{S}_{n}(x)$ has no zeros in $[-1, c]$ we get that $p_{n, p}^{*}$ and $r_{n, p}\left(x, \alpha_{1}, \ldots, \alpha_{n}\right)$ have no zeros in $[-1, c]$ for $1 \leq p<\infty$. Moreover, $r_{n, p}\left(x, \alpha_{1}, \ldots, \alpha_{n}\right)$ uniformly tends to $r_{n, \infty}\left(x, \alpha_{1}, \ldots, \alpha_{n}\right)$ as $p \rightarrow \infty$ which extends the above statement for every $1 \leq p \leq \infty$. This completes the proof the the theorem.

Remark. Since points \pm 1 are oscillation points for $r_{n, \infty}$ it follows immediately from Theorem 5 that $r_{n, \infty}$ has no oscillation points in $(-1, c]$.

Let us also note that by the methods used in [7] it can be easily shown that if we replace the $C$-norm in (1) by the $L_{1}$-norm and assume that $\operatorname{Cl}\left(\bigcup_{n=1}^{\infty} \mathscr{U}_{n}\right)=$ $L_{1}[a, b]$ then $\rho\left(Z\left(T_{n}\right)\right)=O\left(E\left(W^{1}, \mathscr{U}_{n}\right)\right)$ for any solution $T_{n}$ of $(1)$ (the closure of $\mathscr{U}_{n}$ 's and $W^{1}$ are considered for $L_{1}$-norm and $L_{1}$-modulus of continuity, respectively).

\section{REFERENCES}

1. N. I. Achieser, Vorlesungen über Approximations theorie, Akademie Verlag, Berlin, 1953.

2. P. B. Borwein, R. Grothmann, A. Kroó and E. B. Saff, The density of alternation points in rational approximation Proc. Amer. Math. Soc. 105 (1989), 881-888.

3. M. I. Kadec, On the distribution of points of maximal deviation in the approximation of continuous functions by polynomials, Uspekhi Mat. Nauk 15 (1960), 199-202. 
4. S. Karlin and W. J. Studden, Tchebysheff systems: with applications in analysis and statistics, Interscience, New York, 1966.

5. R. C. Jones and L. A. Karlovitz, Equioscillation under nonuniqueness in the approximation of continuous functions, J. Approximation Theory 3 (1970), 138-145.

6. A. Kroó, On the unicity of best Chebyshev approximation of differentiable functions, Acta Sci. Math. (Szeged) 47 (1984), 377-389.

7. A. Kroó and F. Peherstorfer, Interpolatory properties of best $L_{1}$-approximations, Math. Z. 196 (1987), 249-257.

8. _ Interpolatory properties of best rational $L_{1}$-approximations, Constr. Approx. 4 (1988), 97-106.

9. $\ldots$, On the zeros of polynomial of minimal $L_{p}$-norm, Proc. Amer. Math. Soc. 101 (1987), 652-656.

10. G. G. Lorentz, Distribution of alternation points in uniform polynomial approximation, Proc. Amer. Math. Soc. 92 (1984), 401-403.

11. R. Feinerman and D. Newman, Polynomial approximation, Williams-Willins, Baltimore, Md., 1974.

12. E. Passow, Alternating parity of Tchebycheff systems, J. Approximation Theory 9 (1973), 295-298.

13. A. Pinkus and Z. Ziegler, Interlacing properties of the zeros of the error functions in best $L^{p}$-approximations, J. Approximation Theory 27 (1979), 1-18.

14. E. B. Saff, Incomplete and orthogonal polynomials, Approximation Theory IV (C. K. Chui, L. L. Schumaker, and J. D. Ward., eds.), Academic Press, New York, 1983, pp. 219-256.

15. I. Singer, Best approximation in normed linear spaces, Springer, Berlin, Heidelberg, and New York, 1970.

16. Sp. Tashev, On the distribution of points of maximal deviation for the polynomials of best Chebyshev and Hausdorff approximations, Approximation and Function Spaces (Z. Ciesielski, ed.), North-Holland, Amsterdam, 1981, pp. 791-799.

Mathematical Institute of the Hugarian Academy of Sciences, Budapest, Reáltanoda U. 13-15, H-1053, HUNGARY

Institut für MAthematik, J. Kepler Universität LinZ, A-4040 LinZ, Austria 